\title{
Fisioterapia paliativa no adenocarcinoma metastático de pulmão: relato de caso
}

\author{
Palliative physical therapy in metastatic lung adenocarcinoma: case report
}

Fisioterapia paliativa en el adenocarcinoma metastático de pulmón: relato de caso

\section{Recebido: 25/12/2017 \\ Aprovado: 09/05/2018 \\ Publicado: 01/08/2018}

\author{
Jéssica Peixoto Araújo ${ }^{1}$ \\ Almir José Sarri² \\ Deiseane Bonateli ${ }^{3}$ \\ Daniela Santana Polati da Silveira ${ }^{4}$
}

Este é um estudo de caso observacional través de avaliação fisioterapêutica que teve por objetivo avaliar as alterações funcionais do câncer de pulmão no paciente em cuidados paliativos. Participou do estudo uma paciente com adenocarcinoma metastático em cuidados paliativos. Foram identificadas limitações como: redução de amplitude de movimento, diminuição da complacência, retração pulmonar e sintomas relativos ao câncer de pulmão como: dor, dispneia, ansiedade e tristeza. As limitações físicas e psicossociais foram visíveis a partir dos resultados obtidos, comprovando a eficácia da avaliação fisioterapêutica para direcionamento de tratamento, trazendo qualidade de vida à paciente.

Descritores: Cuidados paliativos; Neoplasia pulmonar; Avaliação em saúde.

This is an observational case study made through a physical therapy evaluation that aimed to evaluate the functional changes in lung canser in a patient under palliative care. One patient with metastatic adenocarcinoma under palliative care. Certain limitations were identified, such as: reduction of the amplitude of movement, diminution in complacency, pulmonary retraction and symptoms relative to lung cancer, such as pain, dyspnea, anxiety and sadness. The physical and psychosocial limitations were visible considering the results obtained, making it clear that the physical therapy evaluation was efficient to direct the treatment, bringing quality of life to the patient.

Descriptors: Palliative care; Lung neoplasms; Health evaluation.

Este es un estudio de caso observacional a través de evaluación fisioterapéutica que tuvo como objetivo evaluar las alteraciones funcionales del cáncer de pulmón en el paciente en cuidados paliativos. Participó del estudio una paciente con adenocarcinoma metastático en cuidados paliativos. Fueron identificadas limitaciones como: reducción de amplitud del movimiento, disminución de la complacencia, retracción pulmonar y síntomas relativos al cáncer de pulmón como: dolor, disnea, ansiedad y tristeza. Las limitaciones físicas y psicosociales fueron visibles a partir de los resultados obtenidos, comprobando la eficacia de la evaluación fisioterapéutica para direccionamiento de tratamiento, trayendo calidad de vida al paciente.

Descriptores: Cuidados paliativos; Neoplasia pulmonar; Evaluación en salud.

1. Fisioterapeuta. Integrante do Grupo de Pesquisa Qualidade de Morte do Hospital de Câncer de Barretos, SP, Brasil. ORCID: 0000-0001-7130-1976 E-mail: jpa.fisio@outlook.com

2. Fisioterapeuta. Mestre e Doutor em Ginecologia, Obstetrícia e Mastologia. Coordenador do Departamento de Fisioterapia Oncológica e da Residência em Fisioterapia Oncológica da Fundação Pio XII - Hospital de Câncer de Barretos, SP, Brasil. ORCID: 0000-0001-9184-584X E-mail: almirsarri@hotmail.com

3. Fisioterapeuta. Especialista em Ortopedia e Traumatologia nas Patologias e Cirurgias de Joelho. Fisioterapeuta do Hospital de Câncer de Barretos, SP, Brasil. ORCID: 0000-0002-8564-4408 E-mail: deisebona@hotmail.com

4. Fisioterapeuta. Especialista em Reabilitação Musculoesquelética. Especialista em Fisioterapia em Cancerologia. Mestre em Ciências Médicas. Doutoranda em Reabilitação e Desempenho Funcional pela Faculdade de Medicina de Ribeirão Preto/Universidade de São Paulo (FMRP/USP). Docente do Curso de Fisioterapia na Universidade de Franca, SP, Brasil. ORCID: 0000-0001-7352-8445 E-mail: daniela.silveira@unifran.edu.br 


\section{INTRODUÇÃO}

A nualmente cerca de 11 milhões de pessoas são diagnosticadas com câncer. Acredita-se que, em 2030, o número de novos casos passará de 26 milhões, sendo que 17 milhões serão óbitos. Essa situação se explica devido aos fatores de risco não prevenidos da sociedade, como: tabagismo, alimentação e a falta de atividade física.

O câncer mais frequente na população é a neoplasia de pulmão, desde 1985, ocasionado, na maioria das vezes, pelo tabagismo. 0 câncer de pulmão corresponde a $12 \%$ de todos os cânceres e representa a maior taxa de mortalidade, por ser diagnosticado já em estado avançado. Em consequência de o diagnóstico ser tardio, não se tem um bom prognóstico. Os sintomas mais relevantes são tosse, dispneia, hemoptise e dor torácica1.

0 câncer é descrito como a proliferação de células de forma desordenada e anormal, tendo como característica a perda dos mecanismos que são essenciais para sua sobrevivência e duplicação, ocasionando alterações genéticas que progressivamente transformam uma célula normal em maligna ${ }^{2}$.

Os homens eram os que mais apresentavam câncer de pulmão, por ser a população que mais consumia o cigarro, mas com o passar dos anos, as mulheres tem desenvolvido mais a doença do que os homens. 0 câncer de pulmão pode ser classificado em dois tipos, carcinoma de pequenas células e carcinoma de células não pequenas. 0 mais comum é o de células não pequenas. Existem três tipos histológicos de câncer de pulmão: adenocarcinoma que é responsável por $50 \%$ dos casos, o carcinoma de células escamosas 25 a 30\% dos casos e o de células grandes que corresponde a $15 \%$ dos $\operatorname{casos}^{3,4}$.

Os pacientes com câncer de pulmão sofrem um grande risco de metástases, sendo disseminadas principalmente para os linfonodos, ossos, glândulas adrenais e cérebro. A sobrevida está relacionada à progressão do câncer, ou seja, no estágio III a sobrevivência de cinco anos é 7 a 19\%, já no estágio IV é $2 \%$. Na maioria dos diagnósticos por não se ter mais possibilidades de cura, é indicado o cuidado paliativo. Ele deve ser articulado e programado pela equipe multidisciplinar. 0 conhecimento de várias alternativas paliativas proporciona amenizar os sintomas, e confortar o paciente e sua família ${ }^{1}$.

Os cuidados paliativos proporcionam ao paciente e sua família qualidade de vida, através do alívio da dor e sintomas angustiantes. Utilizam a abordagem multidisciplinar para atender todas as necessidades dos pacientes e familiares, dentro do meio biopsicossocial e espiritual ${ }^{5}$.

Acredita-se que com uma avaliação minuciosa, pode-se melhorar o tratamento fisioterapêutico proposto ajudando o paciente na melhora dos seus principais sinais e sintomas, como por exemplo, a dispneia e a dor, que são os mais frequentes ${ }^{6}$.

A avaliação fisioterapêutica é importante para nortear a intervenção e a evolução clínica do paciente desde o momento do prognóstico até o processo de morte. A avaliação é seguida pela verificação do hemograma, pressão arterial (PA), frequência cardíaca e respiratória (FC e FR), saturação de oxigênio $\left(\mathrm{SatO}_{2}\right)$ e análise do prontuário, buscando realizar os procedimentos necessários de acordo com a condição e a necessidade do paciente ${ }^{6}$.

Este estudo teve por objetivo avaliar as alterações funcionais de uma paciente em cuidados paliativos, decorrentes do câncer de pulmão.

\section{MÉTODO}

Este é um estudo de caso observacional realizado no mês de outubro do ano de 2017 no Hospital de Câncer de Barretos, através de uma avaliação fisioterapêutica, contendo coleta de dados pessoais, exame físico, avaliação do sistema respiratório, suporte ventilatório, avaliação neurológica e, diagnóstico fisioterapêutico com intuito de esclarecer as alterações funcionais decorrentes do adenocarcinoma metastático e seu tratamento.

Foi utilizado um instrumento de avaliação que obteve dados referentes a coleta de dados pessoais, exame físico, avaliação do 
sistema respiratório, suporte ventilatório, avaliação neurológica e diagnóstico fisioterapêutico, com tudo isso foi observado limitações funcionais relativas à redução da amplitude de movimento em membro superior direito (MSD: flexão $116^{\circ}$, extensão $40^{\circ}$, adução $35^{\circ}$, abdução $127^{\circ}$. MSE: flexão $170^{\circ}$, extensão $43^{\circ}$, adução $35^{\circ}$, abdução $165^{\circ}$ ), diminuição da complacência e retração pulmonar, diminuição da força muscular em MSD (comparado com o lado oposto). Para avaliação de sinais e sintomas se utilizou a Escala ESAS $^{8}$.

A coleta de dados foi realizada dentro das normas do Ministério da Saúde e Conselho Nacional de Ética em Pesquisa com embasamento na resolução $\mathrm{n}^{\circ} 196 / 96$ versão 2012, aprovada pelo Comitê de Ética em Pesquisa (CEP) da instituição, o qual foi cadastrado sob o número 1798/2017.

\section{RESULTADOS}

0 caso em pauta é de uma paciente com câncer de pulmão metastático em cuidados paliativos. Paciente SLP, feminino, 48 anos, casada, através de uma consulta relatando: desconforto respiratório constatou-se um derrame pleural e por meio de investigação da broncoscopia na origem, recebeu diagnostico de carcinoma espinocelular (CEC).

Foi encaminhada para o Hospital de tratamento em 14/09/2012 onde foi diagnosticada com Adenocarcinoma Primário do Pulmão esquerdo com estadiamento: T4N3M1a.

Em 06/06/2013, fez ressecção de nodulação em parede torácica direita. Recebeu orientações sobre a impossibilidade de cura e foi encaminhada para os cuidados paliativos em 02/08/2013. Foi proposto tratamento com quimioterapia e radioterapia paliativa e, identificada metástase para o fígado, pleuras, pulmão, sistema nervoso central (SNC), anexos e pericárdio.

As queixas principais: dor no hemitórax direito, dispneia, limitação de ADM de ombro. Realizou exames complementares de tomografia computadorizada, cintilografia óssea, ressonância magnética e exames laboratoriais solicitados no decorrer do tratamento.

0 trabalho oncológico foi realizado pela equipe multidisciplinar diariamente, de acordo com as necessidades específicas da paciente. A fisioterapia seguiu um protocolo com uma conduta de exercícios ativos, para ganho de amplitude de movimento, treino respiratório e alívio dos principais sintomas e também, a eletroterapia para analgesia. Em relação aos sintomas presentes a escala ESAS que avalia os sintomas é apresentada na Tabela 1.

A dispneia representava grau III na escala de dispneia modificada do Medical Research Council (MRC) correspondendo ao item: anda mais devagar do que pessoas da mesma idade devido à falta de ar, ou quando caminha no plano, no próprio passo, para respirar.

Foi relatado pela paciente um grande desconforto referido como dor no hemitoráx direito, principalmente na região do ombro e pescoço, além de outros sintomas.

A avaliação foi realizada com eficácia para diagnóstico de limitações presentes devido ao tratamento e à doença em si. Tais limitações podem ser tratadas pelo fisioterapeuta, propondo um melhor prognóstico funcional, auxiliando na qualidade de vida nos cuidados paliativos. 
Tabela 1. Avaliação dos sintomas (escala ESAS). Barretos, Outubro de 2017.

\begin{tabular}{|c|c|c|c|c|c|c|c|c|c|c|c|c|}
\hline \multirow{5}{*}{$\begin{array}{c}\text { Sem dor } \\
\text { Sem cansaço } \\
\text { (fraqueza) } \\
\text { Sem náusea } \\
\text { (enjoo) }\end{array}$} & 0 & 1 & 2 & 3 & 4 & 5 & 6 & 7 & 8 & 9 & 10 & \multirow{2}{*}{$\begin{array}{c}\text { Pior dor possível } \\
\text { Pior cansaço (fraqueza) }\end{array}$} \\
\hline & & & & & & & & & & & & \\
\hline & 0 & 1 & 2 & 3 & 4 & 5 & 6 & 7 & & 9 & 10 & possível \\
\hline & & & & & & & & & & & & Pior náusea (enjoo) \\
\hline & 0 & 1 & 2 & 3 & 4 & 5 & 6 & 7 & & 9 & 10 & possível \\
\hline Sem tristeza & 0 & 1 & 2 & 3 & 4 & 5 & 6 & 7 & 8 & 9 & & Pior tristeza possível \\
\hline Sem ansiedade & 0 & 1 & 2 & 3 & 4 & 5 & 6 & 7 & 8 & 9 & & Pior ansiedade possível \\
\hline Sem sonolência & 0 & 1 & 2 & 3 & 4 & 5 & & 7 & 8 & 9 & 10 & Pior sonolência possível \\
\hline Melhor apetite & & 1 & 2 & 3 & 4 & 5 & 6 & 7 & 8 & 9 & 10 & Pior apetite possível \\
\hline $\begin{array}{c}\text { Melhor sensação } \\
\text { de bem-estar }\end{array}$ & 0 & 1 & 2 & 3 & 4 & 5 & 6 & 7 & & 9 & 10 & $\begin{array}{c}\text { Pior sensação de mal-estar } \\
\text { possível }\end{array}$ \\
\hline Sem falta de ar & 0 & 1 & 2 & 3 & 4 & 5 & 6 & 7 & & 9 & 10 & Pior falta de ar possível \\
\hline Melhor sono & 0 & 1 & 2 & 3 & 4 & 5 & 6 & 7 & 8 & 9 & 10 & Pior sono possível \\
\hline
\end{tabular}

Fonte: Manfredini, 2014, p $114^{8}$.

\section{DISCUSSÃo}

O Cuidado Paliativo busca aliviar, prevenir ou até mesmo tratar os sintomas desconfortáveis. 0 foco não é somente o paciente, mas também seus familiares, visando à suavização dos sinais e sintomas, suporte espiritual e psicossocial, lembrandose das necessidades, valores, cultura e crenças tanto do paciente quanto de sua família, oferecendo qualidade de vida ou morte ${ }^{8}$.

Os principais sintomas referidos em pacientes com câncer e em cuidados paliativos são: dor, náusea, anorexia, fadiga, dispneia, confusão. Os sintomas do câncer de pulmão começam a aparecer logo na fase inicial da doença comprometendo o tratamento ${ }^{8,9}$.

O cuidado paliativo tem um papel importante, não só nos sinais e sintomas, mas de proporcionar bem-estar e qualidade de vida em pacientes com câncer, seja no aspecto físico, psicossocial ou espiritual ${ }^{10,11}$. A fisioterapia neste momento é fundamental para promover funcionalidade e independência ao paciente para que possa realizar atividades de vida diária dentro de sua capacidade ${ }^{12}$.

Entre os principais sintomas apresentados pelos pacientes em cuidados paliativos a dispneia é o mais presente, ocasionando enorme sofrimento, desconforto, sufoco e fadiga. Este quadro clínico afeta diretamente a capacidade do paciente em realizar suas atividades de vida diária, o tornando cada vez mais dependente de seus familiares e dos profissionais da saúde, que acabam sofrendo junto com o paciente ${ }^{13,14}$.

0 tratamento fisioterapêutico deve estar incluído no cuidado paliativo, tanto quanto a equipe multidisciplinar, buscando maximizar a qualidade de vida e, proporcionando promoção, prevenção, intervenção e habilitação ${ }^{10}$.

Foram observados resultados positivos com a utilização da mobilização precoce, a partir de atividades fisioterapêuticas progressivas ${ }^{15,16}$. A implementação de outras técnicas de fisioterapia também demonstrou resultados interessantes, como por exemplo, as técnicas usadas para alívio da dor, como: massagem, acupuntura, drenagem linfática manual e cinesioterapia ${ }^{17}$.

0 perfil de paciente em cuidados paliativos é de apresentar muitos sintomas e limitações associados a doença e ao 
tratamento agressivo. A avaliação fisioterapêutica direciona o tratamento conforme as necessidades do paciente ${ }^{6,17}$.

\section{CONCLUSÃO}

Os cuidados paliativos cada dia mais estão sendo indicados como proposta de tratamento não para cura, mas para qualidade de vida, englobando vários aspectos. A avaliação fisioterapêutica nos cuidados paliativos foi inteiramente essencial para determinar a melhor conduta a seguir, minimizando o impacto causado pelo câncer.

A conduta proposta teve como principal meta a intervenção na dor e na funcionalidade, que são os principais sintomas presentes, trazendo a paciente melhor qualidade de vida na terminalidade.

Os estudos sobre o tema abordado, ainda são escassos e, precisam ser ampliados. Há importância da avaliação fisioterapêutica para melhorar o direcionamento do tratamento em cuidados paliativos.

\section{REFERÊNCIAS}

1. Farbicka P, Nowicki A. Palliative care in patients with lung cancer. Contemp Oncol. 2013; 17(3):238-45.

2. Lopes A, Chammas R, Iyeyasu H. Oncologia para graduação. 3a ed. São Paulo: Lemar; 2013. 752p.

3. Franceschini J, Jardim JR, Fernandes ALG, Jamnik S, Santoro IL. Relationship between the magnitude of symptoms and the quality of life: a cluster analysis of lung cancer patients in Brazil. J Bras Pneumol. 2013; 39(1):23-31.

4. Araujo AS. Impacto da ressecção pulmonar por câncer de pulmão nos marcadores inflamatórios após um mês de cirurgia [Internet] [dissertação]. Fortaleza: Faculdade de Medicina, Universidade Federal do Ceará; 2014 [citado em: 22 jan 2017]; 109p. Disponível em: http://www.repositorio.ufc.br/bitstream/riu fc/10835/1/2014_dis_asaraujo.pdf

5. Silveira MH, Ciampone MHT, Gutierrez BAO. Percepção da equipe multiprofissional sobre cuidados paliativos. Rev Bras Geriatr Gerontol. 2014; 17(1):7-16.

6. Peres AK, Carvalho ACA, Peixoto MG, Saito EH. Resgate fisioterapêutico para pacientes com comprometimento da função pulmonar e câncer de pulmão. Rev Hosp Univ Pedro Ernesto. 2015; 14 (Supl 1):97-100.

7. Maldaner M, Rech V, Fracasso JI, Sachetti A. Uso de threshold com pressão expiratória em pós-operatório de lobectomia. Saúde Pesqui. 2014; 7(1):91-6.

8. Manfredini LL. Tradução e validação da escala de avaliação de sintomas de Edmonton (ESAS) em pacientes com câncer avançado [dissertação]. Barretos, SP: Fundação Pio XII, Hospital de Câncer de Barretos; 2014. 168p.

9. Ozalevli S. Impact of physiotherapy on patients with advanced lung cancer. Chron Respir Dis. 2013; 10(4):223-32.

10. Girão M, Alves S. Fisioterapia nos cuidados paliativos. Salutis Sci. 2013; 5:34-41.

11. Santana CS, Tamanini G, Fioravanti JP, Souza RC. 0 tratamento fisioterapêutico da dor nos cuidados paliativos. Rev Ling Acad. 2017; 7(6):41-53.

12. Silva SCB, Gúedes MR. Percepções dos acompanhantes de pacientes em estado de terminalidade. REFACS [Internet]. 2017 [citado em: 20 dez 2018]; 5(2):221-7. Disponível em: http://seer.uftm.edu.br/revistaeletronica/in dex.php/refacs/article/view/1790/pdf

13. Ribeiro 0, Cunha M, Duarte J, Ferreira AL, Ferreira AS, Venício D, et al. A segurança do doente em cuidados paliativos: percepção dos profissionais de saúde. Millenium. 2014; 47:173-89.

14. Kock KS, Rocha PAC, Silvestre JCC, Coelho D, Leite KR. Adequações dos dispositivos de oxigenoterapia em enfermaria hospitalar avaliadas por oximetria de pulso e gasometria arterial. Assobrafir ciênc. 2014; 5(1):53-64.

15. Machado AS, Nunes RD, Rezende AAB. Intervenções fisioterapêuticas para mobilizar precocemente os pacientes internados em unidades de terapia intensiva: estudo de revisão. Rev Amazôn Sci Health. 2016; $4(2): 41-6$.

16. Silva IT, Oliveira AA. Efeitos da mobilização precoce em pacientes críticos internados em UTI. Ciênc Desenvol. 2015; 8(2):41-50.

17. Baltieri L, Passos AIM, Galhardo FDM, Roceto LS, Toro IFC. Avaliação pré-operatória da força muscular respiratória, da função 
pulmonar e da capacidade funcional de pacientes submetidos a ressecção pulmonar. ABCS Health Sci. 2015; 40(1):22-7.
CONTRIBUIÇÕES

Todos autores tiveram iguais

contribuições nas várias fases do estudo e na redação do artigo.

\footnotetext{
Como citar este artigo (Vancouver)

Araújo JP, Sarri AJ, Bonateli D, Silveira DSP. Fisioterapia paliativa no adenocarcinoma metastático de pulmão: relato de caso. REFACS [Internet]. 2018 [citado em: inserir dia, mês e ano de acesso]; 6(3): 522-527. Disponível em: inserir link de acesso. DOI: inserir link do DOI.

\section{Como citar este artigo (ABNT)}

ARAÚJO, J. P. et al. Fisioterapia paliativa no adenocarcinoma metastático de pulmão: relato de caso. REFACS, Uberaba, MG, v. 6, n. 3, p. 522-527, 2018. Disponível em: <inserir link de acesso >. Acesso em: inserir dia, mês e ano de acesso. DOI: inserir link do DOI.

\section{Como citar este artigo (APA)}

Araújo, J. P., Sarri, A. J., Bonateli, D., \& Silveira, D. S. P. (2018). Fisioterapia paliativa no adenocarcinoma metastático de pulmão: relato de caso. REFACS, 6(3), 522-527. Recuperado em: inserir dia, mês e ano de acesso de inserir link de acesso. DOI: inserir link do DOI.
} 